\title{
Controlling the optical creation of gold nanoparticles in a PVA matrix by direct laser writing
}

\author{
T. Ritacco \\ tiziana.ritacco@fis.unical.it

\section{Ricciardi} \\ loredana.ricciardi@unical.it \\ M. La Deda \\ massimo.ladeda@unical.it
}

\section{Giocondo \\ michele.giocondo@cnr.it}

\author{
Consiglio Nazionale delle Ricerche, Istituto di Nanotecnologia - Nanotec, UOS di Cosenza Ponte Pietro \\ Bucci Cubo 31/C, 87036 Rende (CS), Italy \\ Università della Calabria - Dipartimento di Fisica Ponte Pietro Bucci Cubo 31/C - 87036 Rende (CS), \\ Italy \\ Consiglio Nazionale delle Ricerche, Istituto di Nanotecnologia - Nanotec, UOS di Cosenza Ponte Pietro \\ Bucci Cubo 31/C, 87036 Rende (CS), Italy \\ Università della Calabria - Dipartimento di Chimica e Tecnologie Chimiche Ponte Pietro Bucci Cubo \\ 14/C, 87036 Rende (CS), Italy \\ Consiglio Nazionale delle Ricerche, Istituto di Nanotecnologia - Nanotec, UOS di Cosenza Ponte Pietro \\ Bucci Cubo 31/C, 87036 Rende (CS), Italy \\ Università della Calabria - Dipartimento di Chimica e Tecnologie Chimiche Ponte Pietro Bucci Cubo \\ 14/C, 87036 Rende (CS), Italy \\ Consiglio Nazionale delle Ricerche, Istituto di Nanotecnologia - Nanotec, UOS di Cosenza Ponte Pietro \\ Bucci Cubo 31/C, 87036 Rende (CS), Italy
}

We report about the study on the physical features of gold nano-particles (GNPS) created by 2-photons photo-reduction Direct Laser Writing in a Poly-Vinyl Alcohol (PVA) matrix doped with $\mathrm{HAuCl}_{4}$. We drop cast a film of the PVA+ $\mathrm{HAuCl}_{4}$ onto a glass substrate, in which we create $1 \mathrm{D}$ gratings made by stripes of GNPS with a single laser sweep. We show that the stripe width increases with the laser power and the exposure time. We also analyse the influence of the exposure time over the created nano-particles size distribution and density and we show that by suitably adjusting the exposure time it is possible to maximize the frequency of a given diameter.

By comparing the experimental results with a polymerization "voxel" model, we are able to evaluate the effective cross section for 2photons absorption of our material.

[DOI: http://dx.doi.org/10.2971/jeos.2016.16008]

Keywords: 2P-photoreduction, direct laser writing, nanofabrication

\section{INTRODUCTION}

2-Photons Direct Laser Writing (2-PDLW) is proving as one of the most powerful techniques for $2 \mathrm{D}$ and $3 \mathrm{D}$ rapid microand nano-fabrication in photosensitive materials. The common scheme for the implementation of the technique is based on a IR fs pulsed laser source with a repetition rate of about $80 \mathrm{MHz}$, allowing the delivering of high doses of energy. The laser beam is then tightly focused by a large N.A. optical system and scanned through the UV-sensitive photoresist that polymerizes by 2-P absorption.

The involved third-order non linear process physics, i.e. the cubic dependence of the imaginary part of the third order adsorption coefficient on the laser intensity and the existence of a threshold for the 2-photons absorption process, restricts the polymerization volume to the very central part of the focus figure; this allows for the achievement of 3D polymeric structures with sub-wavelength details, below the diffraction limit [1].

The 2-PDLW technique has been successfully exploited for the realization of biomimetic materials [2,3], mechanical and pho- tonic materials [4, 5], and optical metamaterials [6]. Nevertheless, beyond the "bare" topographic structuring of 3D objects, more specific applications would require the possibility to get a physical or chemical patterning of the structure on the sub-micrometric scale. Some authors have already reported about the realization of a scaffold for living cells, with differentiated affinities for different parts of the cell, in order to impose a well defined configuration to the latter [7]. In this sense, an important improvement of the 2-PDLW technique would be achieved with the possibility to insert in the structures "bricks" (voxels) of different materials, as fluorescent polymers, quantum dots (QDs), or noble metals nanoparticles. In fact, the possibility to combine different materials in the 3D structure would pave the way to the DLW of composite nanomaterials for advanced optics, in which the dielectric and metallic properties have to be finely tuned over subwavelength distances [8].

During the last decade, some papers have been devoted to the creation of polymer/NPs composites by $2 \mathrm{P}$ photo-reduction of metal salts. Kaneko et al. [9] showed the possibility to op- 
tically induce the creation of gold nanoparticles (GNPs) in a polyvinyl alcohol (PVA) matrix doped with $\mathrm{HAuCl}_{4}$. Other Authors reported about the use of $\mathrm{AgNO}_{3}$ to create silver NPs inside the same polymeric matrix [10].

In this paper, we present a study on the characteristics of the voxel of a gold-doped PVA resist, prepared according to Ref. 9. Our samples are made by sets of single segments, each one obtained with a single laser sweep, on a glass substrate. Upon the exposure, GNPs are created inside the swept segment. We find that the width of each segment depends on the delivered energy dose, as expected. Finally, we develop the sample in distilled water in order to to remove all the PVA from the substrate and to obtain stripes of free GNPs on the glass substrate, on which we perform a basic statistical analysis based on scanning electron microscopy (SEM) images, showing the possibility to control the size and the density of the created GNPs.

\section{MATERIALS AND METHODS}

Our DLW system is equipped with a near-IR $(\lambda=780 \mathrm{~nm})$ Ti:Sapphire laser, with pulse duration of $100 \mathrm{fs}$, repetition rate of $80 \mathrm{MHz}$ and maximum outpower of $180 \mathrm{~mW}$.

The laser beam is focused by a $63 \mathrm{X}, 1.4$ N.A. objective and swept by a galvo scanner through the PVA film, with speeds ranging from 10 to $8.000 \mu \mathrm{m} / \mathrm{s}$.

Gold(III) chloride trihydrate $\left(\mathrm{HAuCl}_{4} \bullet 3 \mathrm{H}_{2} \mathrm{O}\right)$ and polyvinyl alcohol (PVA, Mw: 13,000-23,000 Da) were purchased from Sigma-Aldrich. Spectrofluorimetric grade water was used for the preparation of the aqueous solutions.

The sample was prepared as follows: two solutions were achieved by separately dissolving $1.3 \mathrm{mg}$ of PVA and $9.5 \mathrm{mg}$ of gold salt in $2 \mathrm{~mL}$ of water each. The two solutions were then mixed and maintained under continuous stirring at room temperature for 24 hours. Figure 1 shows the absorption spectra of a dried film of the mixture (blue line), in comparison to the bare PVA (black line) and $\mathrm{HAuCl}_{4}$ (red line), where the sensitivity to our 2PDLW system is demonstrated by the absorption band peaked around @400 nm, due to the absorption of the gold ions in the polymer.

The resist film was drop casted on a glass substrate and left to dry at room temperature for two hours. The obtained film thickness, measured by the AFM profile of a scratch, is in the order of $1,5 \mu \mathrm{m}$. The laser beam was then focused at the interface between the glass substrate and the photoresist and scanned over the surface.

\section{RESULTS AND DISCUSSION}

We printed 1D gratings, made of $30 \mu \mathrm{m}$ long segments with a period of $3 \mu \mathrm{m}$, covering a surface of $30 \times 30 \mu \mathrm{m}^{2}$. We prepared a series of samples, using different values of the laser power (from $2.5 \mathrm{~mW}$ to $22.5 \mathrm{~mW}$ ) and of the scan speed (from 10 $\mu \mathrm{m} / \mathrm{s}$ to $8000 \mu \mathrm{m} / \mathrm{s})$.

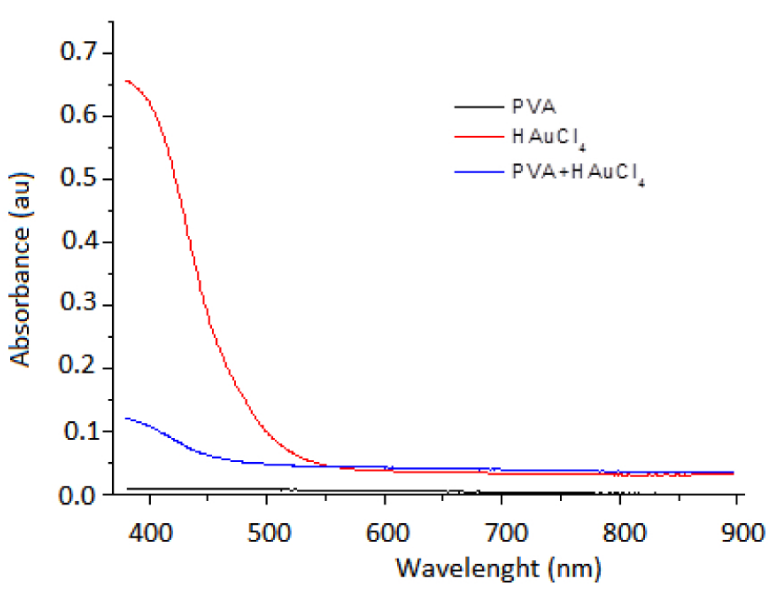

FIG. 1 Absorption spectra of dried films of: gold-doped PVA (blu line), $\mathrm{HAuCl}_{4}$ (red line) and PVA (black line). The absorption band confirms the sensitivity of gold-doped PVA to the $2 \mathrm{P}$ absorption process induced by the $780 \mathrm{~nm}$ laser beam, due to the gold ions.

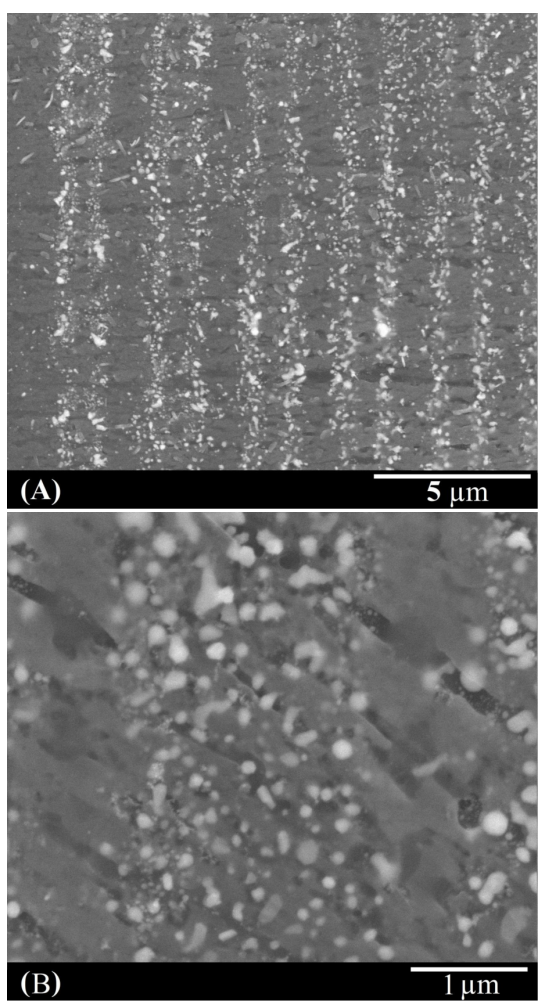

FIG. 2 SEM image of A) a 2-P reduced grating (22.5 mW @ $90 \mu \mathrm{m} / \mathrm{s})$ of GNPs in the PVA matrix; B) a single stripe of the grating rich with GNPs.

$\mathrm{Au}^{(0)}$ nanoparticles were formed inside the scanned stripes, by direct photo-reduction of $\mathrm{AuCl}_{4}^{-}$, according to the mechanism reported in Ref. [11]. An additional reduction mechanism is governed by the thermal effects occurring at temperatures higher than $100^{\circ} \mathrm{C}$, very likely present in our system, because of the local heating induced by the laser [12]-[14].

The width and the characteristics of each stripe was investigated by SEM analyses. Figure 2 shows the SEM images of a sample obtained with $22.5 \mathrm{~mW}$ laser power @90 $\mu \mathrm{m} / \mathrm{s}$ scan speed. The images show the onset of sub-micrometer sized particles inside the exposed areas, embedded in the PVA matrix. 

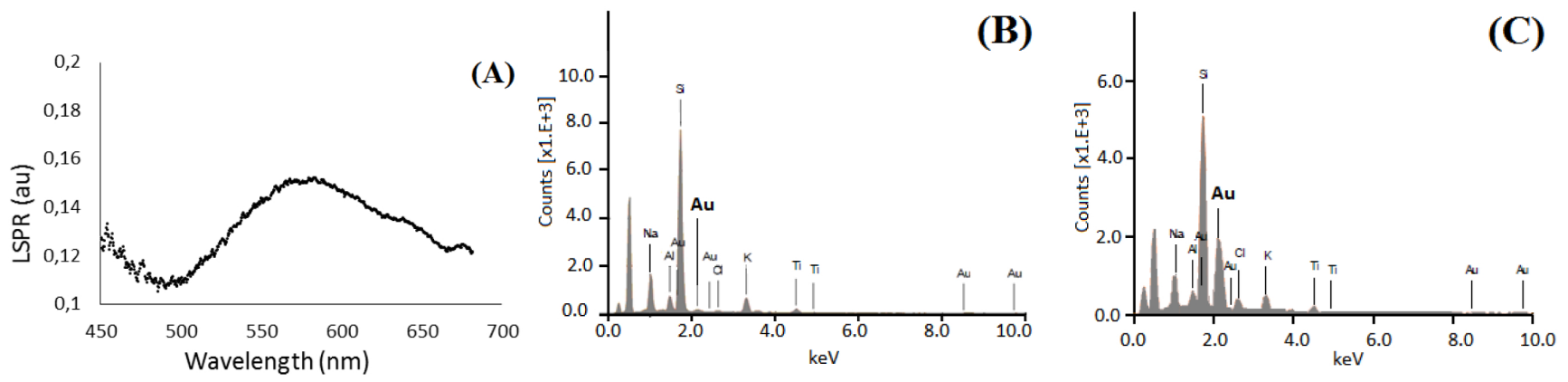

FIG. 3 A) Absorption spectrum performed on the gratings showing the typical localized surface plasmon resonance of gold NPs at @ 570 nm. EDX spectra on the resist before B) and upon $\mathrm{C}$ ) the exposure to the laser beam. The enhancement of the elemental Au peak for the exposed resist demonstrates the nature of the nanoparticles.

The nature of the nanoparticles is revealed by the absorption spectrum (Figure 3(A)), exhibiting the typical gold LSPR absorption band centered at $570 \mathrm{~nm}$. The broadening of the LSPR peak is due to the size dispersion of the GNPs: the diameter of the created nanoparticles, depending on the exposure time and the laser power, varies over two order of magnitude with the largest in the order of $100 \mathrm{~nm}$. Further confirmation is obtained by the back scattering SEM image and by Energy Dispersion X-Ray (EDX) spectra (Figure 3.B-C), performed on the unexposed gold-doped PVA film and on the created segments, showing an important growth of the gold peak in the latter case. Quantitative comparison of the atomic percentages is reported in Table 1.

\begin{tabular}{|c|c|c|}
\hline \multirow{2}{*}{ Element } & \multicolumn{2}{|c|}{ Atomic Percentage (\%) } \\
\cline { 2 - 3 } & Unexposed resist & Exposed resist \\
\hline $\mathrm{Na}$ & 10.45 & 10.78 \\
\hline $\mathrm{Al}$ & 5.69 & 3.44 \\
\hline $\mathrm{Si}$ & 62.22 & 51.98 \\
\hline $\mathrm{Cl}$ & 6.26 & 5.67 \\
\hline $\mathrm{K}$ & 8.78 & 7.55 \\
\hline $\mathrm{Ti}$ & 4.10 & 4.14 \\
\hline $\mathrm{Au}$ & $\mathbf{2 . 5 1}$ & $\mathbf{1 6 . 4 3}$ \\
\hline Total & 100.00 & 100.00 \\
\hline
\end{tabular}

TABLE 1 Atomic percentages of: the glass substrate, the unexposed resist and the created stripes of nanoparticles. The huge presence of gold upon the exposure demonstrates the GNPS creation.

The GNPs are created inside the PVA layer and, therefore, the difference in their brightness is due the polymer screening effect, varying with the z-position occupied by the GNP inside the PVA film, as shown by the SEM images. In fact, the 2$P$ reduction occurs in all the volume irradiated by the laser beam, provided that the intensity overcomes the 2-P absorption threshold, and the GNPs are created at different heights.

The width of the GNPs stripes, as expected because of the 2-P absorption threshold, depends on the power of the irradiating laser beam, larger the power larger the voxel width (Figure $4(\mathrm{~A})$ ).

From the analysis of the power/time dependence of the voxel size, we can derive further details on the physics underlying the observed phenomena. To get this goal, the experimental data are compared with the theoretical predictions on the voxel size reported in Ref [15].
According to this model, the diameter of the voxel generated by the 2-P absorption, depends on the laser power $P$ and on the exposure time $t$, according to the law:

$$
d(P, t)=r_{\omega_{0}} \sqrt{\ln \left(4 \frac{P^{2} t}{v t_{L}\left(r_{\omega_{0}}^{2} \pi \cdot \hbar \omega\right)^{2}} \frac{\sigma_{2}}{\ln \left(\frac{\rho_{0}}{\rho_{0}-\rho_{t h}}\right)}\right)}
$$

Where $r_{\omega_{0}}$ is the laser beam waist, $\hbar \omega$ is the energy of a photon, $v$ is the pulsed laser repetition rate, $t_{L}$ is the single pulse width, $\sigma_{2} / \ln \left[\rho_{0} /\left(\rho_{0}-\rho_{t h}\right)\right]$ is the effective cross section for the 2-P absorption, $\rho_{0}$ and $\rho_{\text {th }}$ are the initial density and the threshold density of radicals respectively. It is important to stress that this model holds for bare photo-sensitive polymers and in our system doesn't occur any polymerization. Therefore, the physical meaning of parameters $\rho$ is different from that exposed above. In particular, the term $\ln \left[\rho_{0} /\left(\rho_{0}-\rho_{t h}\right)\right]$ expressing the polymerization probability, in our case should account for different physical quantities as f.i. the seeds density. As a consequence, we consider only the effective 2photons absorption cross-section, $\sigma_{2}^{*}=\sigma_{2} / \ln \left[\rho_{0} /\left(\rho_{0}-\rho_{t h}\right)\right]$, where $\rho_{0}$ and $\rho_{t h}$ have to be considered in the sense exposed above. Eq. (1) therefore reads:

$$
d(P, t)=r_{\omega_{0}} \sqrt{\ln \left(4 \frac{P^{2} t}{v t_{L}\left(r_{\omega_{0}}^{2} \pi \cdot \hbar \omega\right)^{2}} \sigma_{2}^{*}\right)}
$$

The measured values for the width of the stripes Vs. laser power are then fitted with Eq. (2), in the form

$$
f(x)=A \sqrt{\ln \left(B x^{2} / A^{4}\right)}
$$

where $A=r_{\omega_{0}}$

$B=\frac{4}{v t_{L}(\pi \cdot \hbar \omega)^{2}} \sigma_{2}^{*} t$ where $t$ is expressed by the ratio between $2 r_{\omega_{0}}$ and the scanning speed.

$x=P$.

The constants A and B are the fit parameters. In Figure 4(A) the measured width for stripes written @250 $\mu \mathrm{m} / \mathrm{s}$ Vs. the laser power are compared with the best fit of Eq. (2). Due to the irregular shape of the segments, the experimental data reported are an average value of the measured stripe width and we assumed the dispersion range as uncertainty, considerably larger than the actual measurement uncertainty.

From the fit, we got $r_{\omega_{0}}=(780 \pm 70) \mathrm{nm}$, in the order of the typical beam waist in our system. This should be the asymptotic value for the voxel width when $P$ or $t$ diverge. 


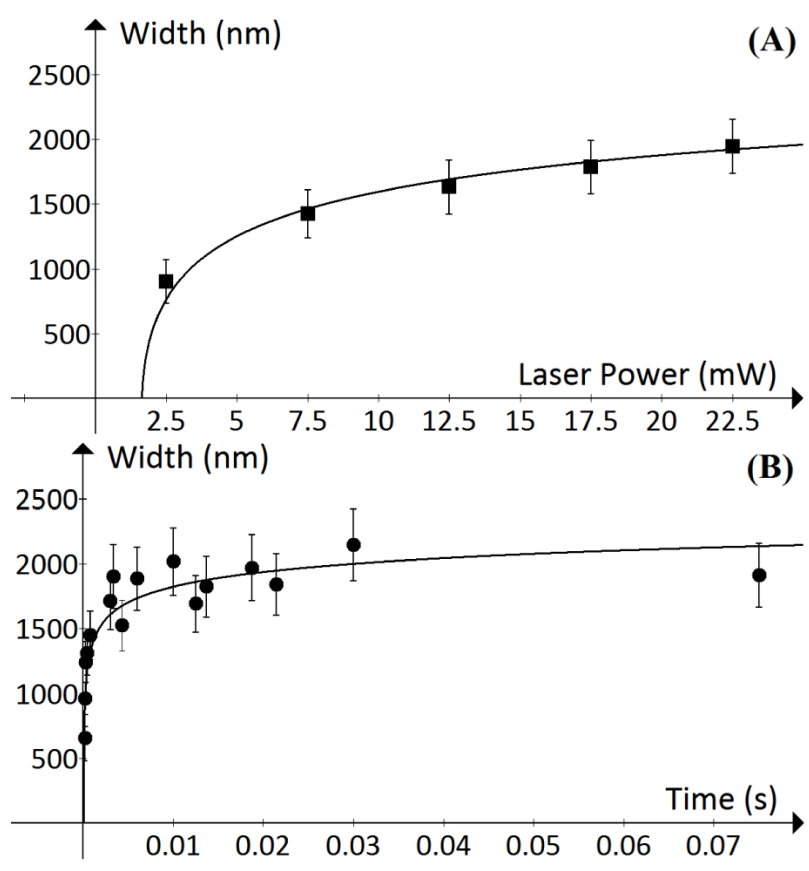

FIG. 4 Dependency of the width of the stripes on A) on the laser power at a fixed scan velocity $250 \mu \mathrm{m} / \mathrm{s}$ and $\mathbf{B}$ ) on the exposure time at a fixed value of laser power of $17.5 \mathrm{~mW}$. Dots are the experimental data and the solid line is the best fit of Eq. (2)

On the other hand, the typical GNPs stripes width actually measured for large values of $P$ and $t$ is even larger; this evidence suggests that the role played by the thermal effect could not be negligible in the phenomenology, extending the region where the gold reduction takes place. Anyhow, the detailed analysis of the thermal effects goes beyond of the spirit of the present paper.

From the obtained B parameter we got the effective cross section $\sigma_{2}^{*}=(3.9 \pm 0.5) 10^{-51} \mathrm{~cm}^{4} \mathrm{~s}$, comparable to those reported for photo-sensitive polymers [15]. This parameter characterizes the "sensitivity" of the material and should be taken into account if hybrid systems are considered, in the case one aims to embed GNPs in polymeric 3D structures.

We also performed an analysis of the NPs diameter as a function of the exposure time, at fixed values of the laser power. In Figure 4(B) are reported the measured segment width data obtained @17.5 mW and the best fit of Eq. (2), from which we got identical values for $\sigma_{2}^{*}=(3.5 \pm 0.7) 10^{-51} \mathrm{~cm}^{4} \mathrm{~s}$ and $r_{\omega_{0}}=(740 \pm 60) \mathrm{nm}$.

The sample was then developed in distilled water for 20 minutes and left to dry at room temperature over a few hours. SEM imaging reveals that after the development the PVA was completely removed and the gratings were made by the solely gold nanoparticles sticking on the glass substrate (Figure 5(A)). On the water developed samples, we performed a basic statistical analysis on the NPs size and density, based on the SEM images. By analysing several gratings created at fixed laser power and different scan speed values, we found a density of GNPs ranging from $90 \mu \mathrm{m}^{-2}$ to $360 \mu \mathrm{m}^{-2}$, increasing with the exposure time.

The results of this analysis are summarized in the histogram of
Figure 5(B), showing that the growth of GNPs with a diameter of about $10 \mathrm{~nm}$ is highly favourite when the exposure time increases.

This behaviour can be qualitatively explained considering that the $\mathrm{AuCl}_{4}^{-}$ions are mostly evenly distributed inside the PVA matrix, but one can expect the presence of localized density peaks. After the reduction onset, the GNPs growth is governed by a diffusive process, favouring at short times the growth of larger particles in the areas where the local ion density is higher. The remaining exposed areas, where the ions are more uniformly distributed, produce smaller GNPs because of the slower growth rate due to the smaller ions density and therefore the process lasts longer. The created GNPs size is finally governed by the growth process depending on the $\mathrm{Au}^{(I I I)}$ ions density and on their characteristic diffusion time inside the PVA matrix. A further factor is given by the local heating, that could affect the diffusion constant.

We don't see any considerable variation in the distribution with the investigated scan speeds for GNPs larger than 50nm: the analysis has confirmed a density of $(45 \pm 5) \mu \mathrm{m}^{-2}$ for all the studied cases. Therefore, the average diameter of the free GNPs varies from $40 \mathrm{~nm}$ obtained at the highest scan speed, to $17 \mathrm{~nm}$ obtained at the lowest one. SEM images of the gratings (Figure 5(C)-(E)) confirm these results: decreasing the velocity of the process, increases the number of small GNPs.

\section{CONCLUSIONS}

We carried out a study on the voxel characterization of a gold ion-doped PVA photoresist. By printing through 2-PDLW in the dry films of resist, we are able to create gratings whose lines are rich in GNPs. We demonstrate that the stripes width depends on the laser power and on the exposure time, and the system behaves according the 2-P absorption voxel model, from which we can evaluate the effective 2-P absorption crosssection correlated to the seeding probability.

A study on the size and the density of the free GNPs of a water developed sample as a function of the exposure time was also carried out: by increasing the exposure time, we observe an enhancement of the probability to create nanoparticles with a diameter $<50 \mathrm{~nm}$.

Therefore, by controlling the energy delivered on the photoresist, we are able to modulate the voxel and the average size of the created GNPs, along with their density. This study could be useful for defining the most well suited DLW printing parameters in the case a polymeric/GNPs nanocomposite is required.

\section{ACKNOWLEDGEMENTS}

The experiments have been performed at Beyond-Nano Cosenza - CNR User Facility. We acknowledge the experimental support of Dr. Franco Cofone, Dr. Giovanni Desiserio, Dr. Irina Gryn and Dr. Mariano Davoli. 

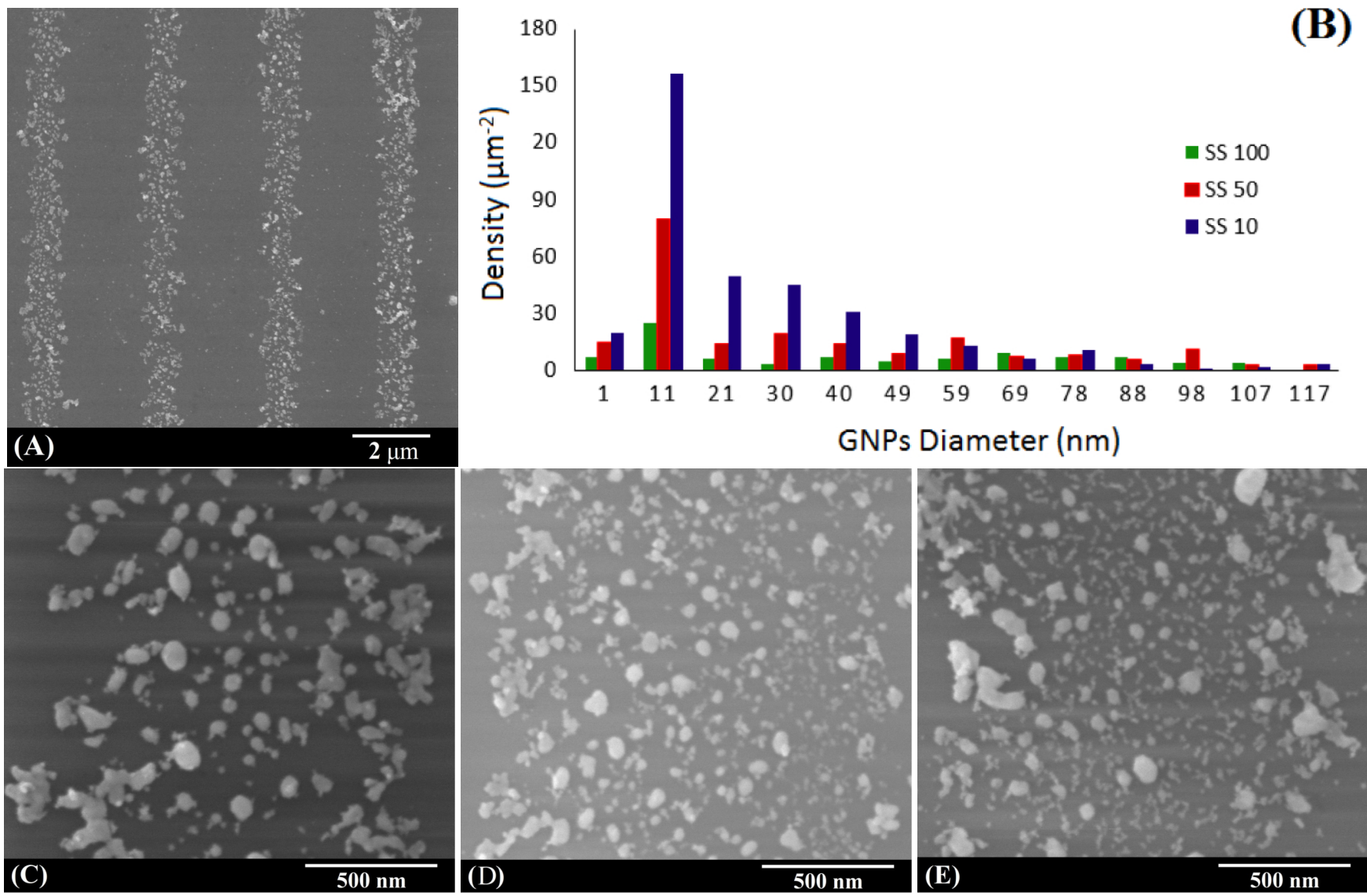

FIG. 5 A) SEM image of a grating (2.5 mW @ $100 \mu \mathrm{m} / \mathrm{s}$ ) of gold nanoparticles free on the glass, after the water development process. B) Histogram of the density of nanoparticles in function of their diameter, for gratings printed at $100 \mu \mathrm{m} / \mathrm{s}$ (C), $50 \mu \mathrm{m} / \mathrm{s}$ (D) and $10 \mu \mathrm{m} / \mathrm{s}$ (E), at a laser power of $12.5 \mathrm{~mW}$. SEM images C-E, of stripes for each gratings, show the rising number of GNPs about $10 \mathrm{~nm}$ at the decreasing of the scan speed.

\section{References}

[1] S. Engelhardt, "Direct Laser Writing," in Laser Technology in Biomimetics, V. Schmidt, and M. R. Belegratis, eds., 13-56 (Springer-Verlag, Berlin/Heidelberg, 2013).

[2] M. Roehrig, M. Thiel, M. Worgull, and H. Hölscher, " $3 \mathrm{D}$ Direct Laser Writing of Nano- and Microstructured Hierarchical GeckoMimicking Surfaces," Small 8(19), 3009-3015 (2012).

[3] W. Barthlott, T. Schimmel, S. Wiersch, K. Koch, M. Brede, M. Barczewski , S. Walheim, et al., "The Salvinia Paradox: Superhydrophobic Surfaces with Hydrophilic Pins for Air Retention Under Water," Adv. Mater. 22 (21), 2325-2328 (2010).

[4] T. Bueckmann, N. Stenger, M. Kadic, J. Kaschke, A. Frölich, T. Kennerknecht, C. Eberl, et al., "Tailored 3D Mechanical Metamaterials Made by Dip-in Direct-Laser-Writing Optical Lithography," Adv. Mater. 24(20), 2710-2714 (2012).

[5] L. Yuan, M. L. Ng, and P. R. Herman, “Femtosecond laser writing of phase-tuned volume gratings for symmetry control in 3D photonic crystal holographic lithography," Optical Materials Express 5(3), 515-529 (2015).

[6] T. Ergin, N. Stenger, P. Brenner, J. Pendry, and M. Wegener, "ThreeDimensional Invisibility Cloak at Optical Wavelengths," Science 328, 337-339 (2010).

[7] B. Richter, T. Pauloehrl, J. Kaschke, D. Fichtner, J. Fischer, A. M.Greiner, D. Wedlich, et al, "Three-Dimensional Microscaffolds Exhibiting Spatially Resolved Surface Chemistry," Adv. Mater. 25(42), 6117-6122 (2013).
[8] M. Infusino, A. De Luca, A. Veltri, A. Veltri, C. Vazquez-Vazquez, M. A. Correa-Duarte, R. Dhama, et al., "Loss-Mitigated Collective Resonances in Gain-Assisted Plasmonic Mesocapsules," ACS Photonics 1(4), 371-376 (2014).

[9] K. Kaneko, H. B. Sun, X. M. Duan, and S. Kawata, "Two-photon photoreduction of metallic nanoparticle gratings in a polymer matrix," Appl. Phys. Lett. 83(7), 1426-1428 (2003).

[10] R. Kuladeep, L. Jyothi, S. Chakradhar, and D. N. Rao, "Fabrication of metal nanostructures in a polymer matrix using femtosecond laser writing technique," Opt. Eng. 53(7), 071823 (2014).

[11] M. Sakamoto, M. Fujistuka, and T. Majima, "Light as a construction tool of metal nanoparticles: Synthesis and mechanism," J. Photoch. Photobio. C 10, 33-56 (2009).

[12] W. M. Huang, and J. L. Shi, "Synthesis and properties of $\mathrm{ZrO}(2)$ films dispersed with Au nanoparticles," J. Sol-Gel Sci. Techn. 20, 145-151 (2001).

[13] R. Zanella, and C. Louis, "Influence of the conditions of thermal treatments and of storage on the size of the gold particles in Au/Ti02 samples," Catal. Today 107(8), 768-777 (2005).

[14] M. I. A. Umar, C. C. Yap, R. Awang, A. A. Umar, M. M. Salleh, and M. Yahaya, "Formation of gold-coated multilayer graphene via thermal reduction," Mater. Lett. 106, 200-203 (2013).

[15] S. Passinger, Two-Photon Polymerization and Application to Surface Plasmon Polaritons (Cuvillier Verlag, Göttingen, 2008). 